\title{
Unity from conflict
}

\section{Could the evolution of multicellular life have been fuelled by conflict among selective forces acting at different levels of organization?}

\section{Paul B. Rainey}

The transition from unicellular to multicellular life brought with it division of labour, development and a panoply of animal and plant forms. But despite its pivotal role in the history of life, the evolution of multicellularity is poorly understood. Watching the de novo emergence of primordial groups in bacterial populations in the lab has led me to think about mechanisms by which higher levels of complexity could emerge.

In multicellular organisms, lower-level entities (cells) have relinquished their ability to reproduce as independent units and instead replicate exclusively as part of the larger whole. The challenge for biologists is to explain how a population of cells becomes a single entity capable of selfreproduction; that is, how an evolutionary transition that involves a shift in the level at which selection operates, from individual entities to groups of entities, can occur.

Existing theory predicts a central role for cooperation. This prediction is borne out by experiments using bacterial populations in which the evolution of simple undifferentiated groups has been observed. Groups arise from mutant cells that overproduce a cell-cell glue; the failure of cells to separate at cell division leads to the formation of a surface-colonizing mat. The emergence of these mats - which form at the air-liquid interface, an environment that is replete in oxygen - is a triumph of selection acting at the higher level. Glue production is costly to individual cells, but the trait spreads because the group reaps a reward (oxygen) that is denied to cells within the broth.

Although the evolutionary emergence of groups is itself a profound event, such groups are typically short-lived. The reason is simple: selection continues to act at the level of individual cells. In so doing, selection favours mutant types that cheat; that is, cells that no longer produce the glue, but nonetheless take advantage of the benefit that accrues from being part of the group. In the absence of any mechanism to repress or punish them, cheaters prosper - ultimately weakening the fabric of the mat to the point where it collapses.

Mechanisms that repress cheaters are thus integral to the success of multicellular organisms, but their evolution is a paradox. Cheater control is unlikely to evolve in the absence of selection acting strongly at the group level. The catch-22 is that selection is powerless to act at the group level because newly emerged groups are incapable of differential reproduction. Although individual microbes within the group continue to divide, groups as a whole are not able to produce offspring; they are, like soma (the non-reproductive cells of a multicellular organism), an evolutionary dead-end.

What, then, represents a plausible route to multicellularity? Cooperation is a necessary innovation but it is not the only one. Also required is group reproduction and a way to minimize the disruptive effects of cheats. Moreover, these three major events need to occur more or less simultaneously.

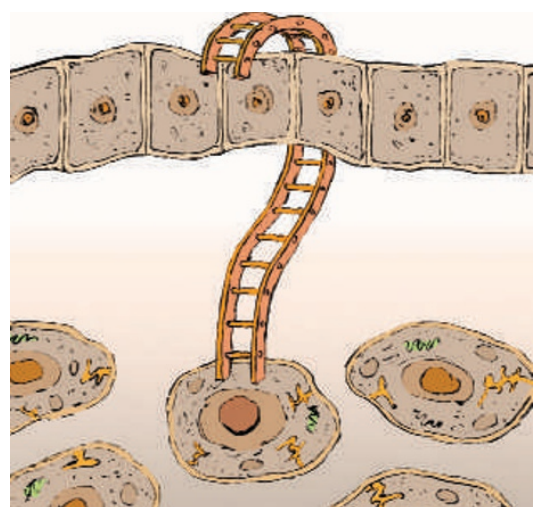

Although the emergence of these three traits in quick succession seems an impossibly difficult challenge for evolution, alternative scenarios in which multicellularity arises in the absence of higher-level selection simply don't make sense. What follows is one plausible scenario.

Evolutionists are increasingly aware of the importance of a multilevel-selection perspective - a way of making sense of the effects of selection at different levels of biological organization. Integral to this perspective is the recognition that processes occurring at different levels (for example at the level of the cell or of the organism), and at different times, are connected: selection at the higher level has consequences for the lower level and vice versa. Although the consequences can be negative, it is just possible that conflicts between selective forces might fuel, rather than hinder, the transition to higher levels of complexity.

Being unable to replicate itself, a newly emerged group of cells is analogous to the soma. The cheater, however, is loosely analogous to a primordial germ line. Selection therefore favours the evolution of cheats, which although destructive to the group, may act as the seeds for the emergence of new groups. In fact, in our experimental populations the cheats are able to swim away from the group. Moreover, because the number of cheats (germ cells) that a group leaves depends on the rate at which cheats arise, selection acting at the higher 'group' level will optimize the rate at which cheats evolve.

Imagine one group in which the mutation rate to cheat is high and another where the rate is low. A group comprised of few cooperating cells will rapidly be destroyed by cheats; moreover, once the group is destroyed, cheats no longer prosper. So the group that has a high rate of cheating will leave few offspring relative to a group that manages to produce a substantial grouplevel structure before cheats arise. Selection acting on the rate of cheating thus becomes a mechanism for cheater control. More significantly though, by equating cheats with germ cells, primordial groups are no longer an evolutionary dead-end. Selection can act at the higher level, but the process occurs via effects wrought at the lower level.

Implicit in this model is a capacity for the cheat to repeatedly switch between groupforming and selfish types by compensatory mutation. This may not always be possible, but in our populations of microbes this capacity does exist. Clearly, little progress towards a more complex group can occur without the eventual evolution of a developmentally regulated mechanism for switching between group and cheat (soma and germ line). But this is by no means beyond the capacity of evolution - given an appropriate selective environment.

Paul B. Rainey is at the School of Biological Sciences, University of Auckland, Private Bag 92019, Auckland, New Zealand.

FURTHER READING

Maynard Smith, J. \& Szathmáry, E. The Major Transitions in Evolution (Freeman, Oxford, 1995).

Michod, R. E. Darwinian Dynamics: Evolutionary Transitions in Fitness and Individuality (Princeton Univ. Press, 1999). Okasha, S. Evolution and the Levels of Selection (Oxford Univ. Press, 2006).

Rainey, P. B. \& Rainey, K. Nature 425, 72-74 (2003).

For other essays in this series, see http:// nature.com/nature/focus/arts/connections/ index.html 